\title{
Secondary Neutron Dose in Carbon-ion Radiotherapy: Investigations in QST-NIRS
}

\author{
Shunsuke Yonai, Shinnosuke Matsumoto \\ Department of Accelerator and Medical Physics, National Institute of Radiological Sciences, Quantum Medical Science Directorate, National Institutes for \\ Quantum and Radiological Science and Technology, Chiba, Japan
}

\section{Review}

Received November 14, 2020

Revision January 21, 2021

Accepted February 4, 2021

Corresponding author: Shunsuke Yonai

National Institutes for Quantum and Radiological Science and Technology, 4-9-1 Anagawa Inage-ku, Chiba 263-8555, Japan

E-mail:yonai.shunsuke@qst.go.jp

(Di) https://orcid.org/0000-0001-9539-7597

This paper is an invited paper recommended by the Japan Health Physics Society (JHPS).

This is an open-access article distributed under the terms of the Creative Commons Attribution License (http://creativecommons.org/licenses/by-nc/4.0), which permits unrestricted use, distribution, and reproduction in any medium, provided the original work is properly cited.

Copyright $\odot 2021$ The Korean Association for Radiation Protection

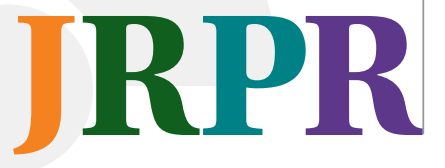

Background: The National Institutes for Quantum and Radiological Science and TechnologyNational Institute of Radiological Sciences (QST-NIRS) has continuously investigated the undesired radiation exposure in ion beam radiotherapy mainly in carbon-ion radiotherapy (CIRT). This review introduces our investigations on the secondary neutron dose in CIRT with the broad and scanning beam methods.

Materials and Methods: The neutron ambient dose equivalents in CIRT are evaluated based on rem meter (WENDI-II) measurements. The out-of-field organ doses assuming prostate cancer and pediatric brain tumor treatments are also evaluated through the Monte Carlo simulation. This evaluation of the out-of-field dose includes contributions from secondary neutrons and secondary charged particles.

Results and Discussion: The measurements of the neutron ambient dose equivalents at a $90^{\circ}$ angle to the beam axis in CIRT with the broad beam method show that the neutron dose per treatment dose in CIRT is lower than that in proton radiotherapy (PRT). For the scanning beam with the energy scanning technique, the neutron dose per treatment dose in CIRT is lower than that in PRT. Moreover, the out-of-field organ doses in CIRT decreased with distance to the target and are less than the lower bound in intensity-modulated radiotherapy (IMRT) shown in AAPM TG-158 (American Association of Physicists in Medicine Task Group).

Conclusion: The evaluation of the out-of-field doses is important from the viewpoint of secondary cancer risk after radiotherapy. Secondary neutrons are the major source in CIRT, especially in the distant area from the target volume. However, the dose level in CIRT is similar or lower than that in PRT and IMRT, even if the contributions from all radiation species are included in the evaluation.

Keywords: Carbon-ion Radiotherapy, Neutron, Out-of-Field Dose, WENDI-II, PHITS

\section{Introduction}

Ion beam radiotherapy (IBRT), including proton radiotherapy (PRT) and carbon-ion radiotherapy (CIRT), can greatly reduce the entrance dose because of the physical characteristics of charged particles, called the Bragg peak. In addition, CIRT offers physical and biological advantages over proton therapy, such as a lower scattering power in medium and a higher biological effect caused by the high linear energy transfer (LET). These physical and biological advantages lead to high-dose localization in the target volume, sparing the surrounding normal tissue and indicating favorable outcomes and shorter treatment duration compared to that of photon and proton radiotherapies [1-5]. 
Meanwhile, the carbon-ion beam can inevitably produce secondary neutrons through nuclear interactions with beamline devices or the patient's body, as well as the proton beam. The secondary neutrons are widely distributed in theory and cause the whole-body exposure of the patient. The impact of secondary neutrons in IBRT on secondary cancer risk is still being discussed due to large uncertainties in the radiationinduced secondary cancer risk, especially for modern treatment modalities, such as intensity-modulated radiotherapy (IMRT) and IBRT [2, 6-9]. However, until now, a number of studies on the dose assessment of the secondary neutron dose in IBRT have been done based on measurements and Monte Carlo simulations, showing the characteristics of secondary neutrons in IBRT in terms of both quantity and quality [10-12].

The National Institutes for Quantum and Radiological Science and Technology-National Institute of Radiological Sciences (QST-NIRS) has continuously investigated the undesired radiation exposure in IBRT, mainly in CIRT [13-19]. The measurement of the secondary neutron dose in the CIRT treatment room had not yet been performed when we started our studies. Therefore, we have provided the measured neutron doses in the CIRT treatment room for several beam delivery system along with the progress of the system ahead of other works. In addition, we had performed investigations on the out-of-field dose and radiation quality in the patient received CIRT by measurement and simulation verified with measured results. Our results on the secondary neutron dose in CIRT and comparisons of these results with other radiation treatment modalities are introduced in this review.

The secondary neutron dose in IBRT is known to depend on the beam delivery method: broad (passive method) and scanning beam methods. The scanning beam method can reduce the production of secondary neutrons because few beamline devices, in which secondary neutrons can be produced, are employed, in which secondary neutrons can be produced, are not employed, implying that the beam efficiency is almost $100 \%$. Neutrons produced in beam-limiting devices, such as multi-leaf and patient-specific collimators, particularly have the most impact on the patient dose in the broad beam method because they are close to the patient, and most primary carbon ions stop in them [15]. Also, a rangeshifter employed depending on the scanning technique can induce interactions with primary beam and produce secondary neutrons.
Therefore, we will show herein the difference of the neutron doses among the beam delivery methods based on rem meter (WENDI-II; Ludlum Measurements Inc., Sweetwater, TX, USA [20]) measurements first [13, 16, 19]. Next, studies on the dose estimation of out-of-field organs will be introduced assuming prostate cancer and pediatric brain tumor treatments with the Monte Carlo simulation $[17,18]$. The out-of-field dose in CIRT is actually derived from secondary neutrons and secondary charged particles. We will present the estimated partial contributions of each particle to the total dose and the doses in out-of-field organs.

\section{Materials and Methods}

\section{Measurement of the Neutron Ambient Dose Equivalent in the CIRT Treatment Room}

Many studies investigating the secondary neutron exposure in PRT were published; however, their results varied by a factor of 100 [10-12]. This variation was mainly derived from different experimental settings, including beam parameters, as noted in detail in [12]. Therefore, we measured the neutron dose in CIRT with various beam delivery methods having the same beam parameters and dosimetry system. The measurements of the neutron dose in PRT with the broad beam method were performed with beam parameters similar to those in CIRT. In contrast, the measurements of the neutron dose in PRT with the scanning beam method were conducted with the beam parameters representing the same target volume and the same maximum target depth with those used in a published study [21].

\section{1) Beam delivery method}

At present, numerous new CIRT treatment facilities are introducing the scanning beam method rather than the broad beam method because of its high irradiation accuracy, flexible treatment planning, and less unwanted dose [22, 23]. At QST-NIRS, the broad beam method was employed for clinical use between 1994 and 2017, and treatments completely shifted to those with the scanning beam method. However, the broad beam method has the highest market share both in CIRT and PRT when our study was started and is still being employed in several facilities; hence, this review presents measurements for both broad and scanning beam methods. In the scanning beam method, in which a pencil beam is scanned transversely by a pair of scanning magnets to cover the target volume, three techniques can be used to 
Table 1. Beam Parameters

\begin{tabular}{lcc}
\hline No. & Max. beam energy $\left(\mathrm{MeV} \cdot \mathrm{u}^{-1}\right)$ & Irradiation volume $\left(\mathrm{mm}^{3}\right)$ \\
\hline 1 & 290 & $58 \times 50 \times 60$ \\
2 & 400 & $58 \times 50 \times 60$ \\
3 & 350 & $100 \times 100 \times 100$
\end{tabular}

achieve the depth-dose distribution in the target volume: range shifter scanning, energy scanning, and hybrid depth scanning. In the range shifter and energy scanning techniques, a pristine Bragg peak by a mono-energetic ion beam is shifted using energy degraders (e.g., range shifters) and by changing the beam energy extracted from an accelerator, respectively. The hybrid scanning technique combines the range shifter and energy scanning techniques. For clinical use at the QST-NIRS, the range shifter and hybrid scanning techniques were employed in 2011 and from 2012 to 2015, respectively. The energy scanning technique has been employed since September 2015 [24].

\section{2) Beam parameter}

Table 1 shows the beam parameters used in the measurements in this review. Nos. 1 and 2 were continuously used in our systematic measurements. No. 3 was used for a comparison with those in PRT with the scanning beam method, in which the $350 \mathrm{MeV} \cdot \mathrm{u}^{-1}$ carbon-ion beam can achieve a range similar to that of the $177 \mathrm{MeV}$ proton beam used in [21].

\section{3) Dosimeter}

The neutron ambient dose equivalent $\mathrm{H}^{*}(10)$ was measured using WENDI-II, a rem meter with a response extended to $5 \mathrm{GeV}$ neutrons [20]. Extended-range rem meters are one of the potential detectors for assessing neutron non-primary radiation required for the basic safety and essential performance of medical electrical equipment by the International Electrotechnical Commission [25].

\section{4) Experimental geometry}

Fig. 1 displays a schematic view of the experimental setup. The center of a water phantom (external size: $22 \times 24 \times 39 \mathrm{~cm}^{3}$ ) was set to be coincidental with the isocenter (IC). The IC was $125 \mathrm{~cm}$-high. WENDI-II was located at distances $d$ of 50, 100, 150 , and 173, or $200 \mathrm{~cm}$ from the position of IC and on the IC line orthogonal to the beam axis. We measured these less than $60^{\circ}$ and $120^{\circ}$ angles with respect to the beam for the energy scanning technique to show the angular dependency, but are not discussed herein.

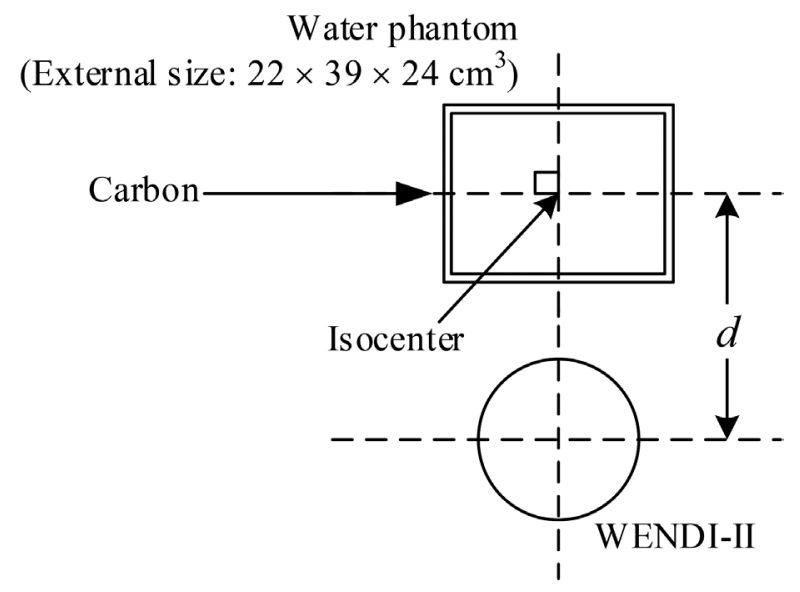

Fig. 1. Schematic view of the experimental setup.

2. Monte Carlo Calculations of the Out-of-Field Organ Doses in CIRT Assuming Prostate Cancer and Pediatric Brain Tumor Treatments

1) Monte Carlo simulation

The particle and heavy ion transport code system (PHITS) was used to calculate the out-of-field organ doses in our studies [26]. The Heavy Ion Medical Accelerator in Chiba (HIMAC) beamline devices of the broad beam method at the QST-NIRS were simulated as the calculation geometry. The reference phantom (male) in the International Commission on Radiological Protection (ICRP) Publication 110 [27] was used as an anthropomorphic phantom to estimate the organ doses during CIRT for the prostate cancer treatment and the computational phantom of a 5-year-old girl developed at the University of Florida [28] for pediatric brain tumor (cerebellar ependymoma) treatment. The ICRP reference phantom (female) was also used to compare the organ doses in adult and pediatric phantoms during CIRT for brain tumor.

We evaluated the dose-averaged dose equivalent based on the calculated organ absorbed dose and the dose-averaged quality factor with the Q-LET relationship based on the ICRP 60 recommendation [29].

Two types of secondary neutron can be found in IBRT: one is an external neutron produced in irradiation devices, such as collimators, and the other is an internal neutron produced in a patient. In theory, when the scanning beam method is employed, the external neutrons are significantly less than those in the broad beam method because beam-limiting devices (e.g., collimators) are not employed. In other words, the internal neutrons in IBRT with the broad beam method are almost the same with the secondary neutrons in IBRT with the scanning beam method using the energy scanning tech- 
nique. Therefore, we used the PHITS counter function to identify the cell in which the secondary neutron was produced and to investigate the partial contributions of the external and internal neutrons to the total dose.

\section{2) Beam parameter}

The beam parameters for the prostate cancer treatment were determined from the typical beam parameters for the same treatment at HIMAC. The detailed parameters are shown in [17], but the typical fractions were 13 fractions from the opposite horizontal $400 \mathrm{MeV} \cdot \mathrm{u}^{-1}$ beams and three fractions from one vertical $290 \mathrm{MeV} \cdot \mathrm{u}^{-1}$ beam assuming that the total prescribed dose was $57.6 \mathrm{~Gy}$ (relative biological effectiveness [RBE]), and the fractionation number was 16. Mean-

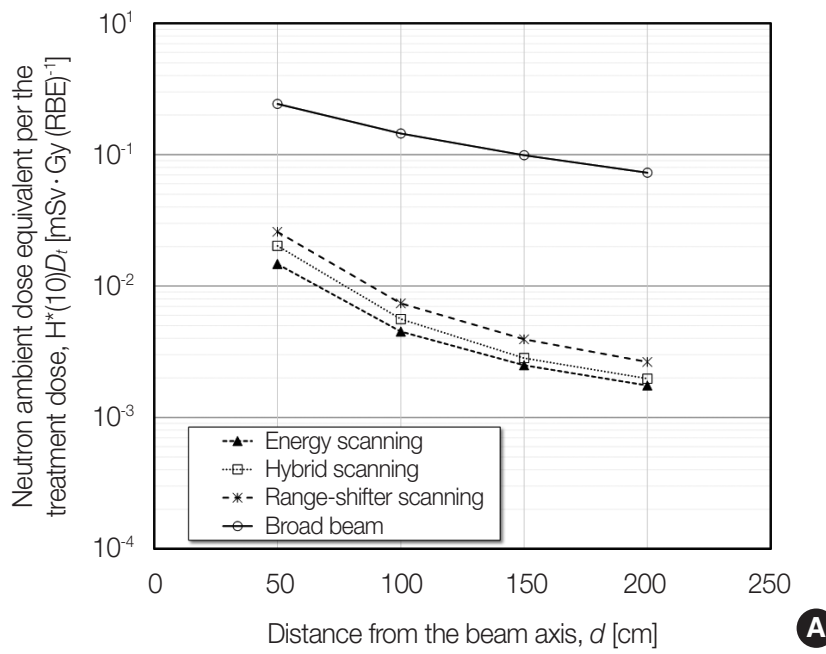

while, the beam parameters for the pediatric brain tumor treatment were determined assuming a sphere with $5.6 \mathrm{~cm}$ diameter in the center of the cerebellum as a typical pediatric cerebellar ependymoma. The beam energy was set to 290 $\mathrm{MeV} \cdot \mathrm{u}^{-1}$ to achieve sufficient length to cover the target. In addition, the beam direction was assumed to be two opposite horizontal irradiations.

\section{Results and Discussion}

\section{Measurement of the Neutron Ambient Dose Equivalent in the CIRT Treatment Room}

1) Measured neutron ambient dose equivalent

Fig. 2 shows the measured neutron ambient dose equiva-

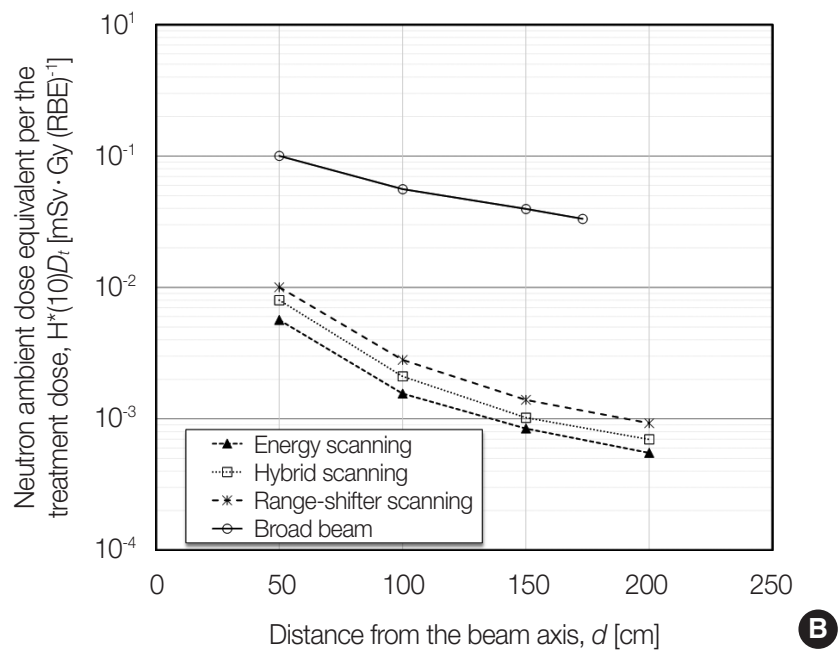

Fig. 2. Measured neutron ambient dose equivalent in carbon-ion radiotherapy with various beam delivery methods: $(A) 400 \mathrm{MeV} \cdot \mathrm{u}^{-1}$ and $(\mathrm{B})$ $290 \mathrm{MeV} \cdot \mathrm{u}^{-1}$.
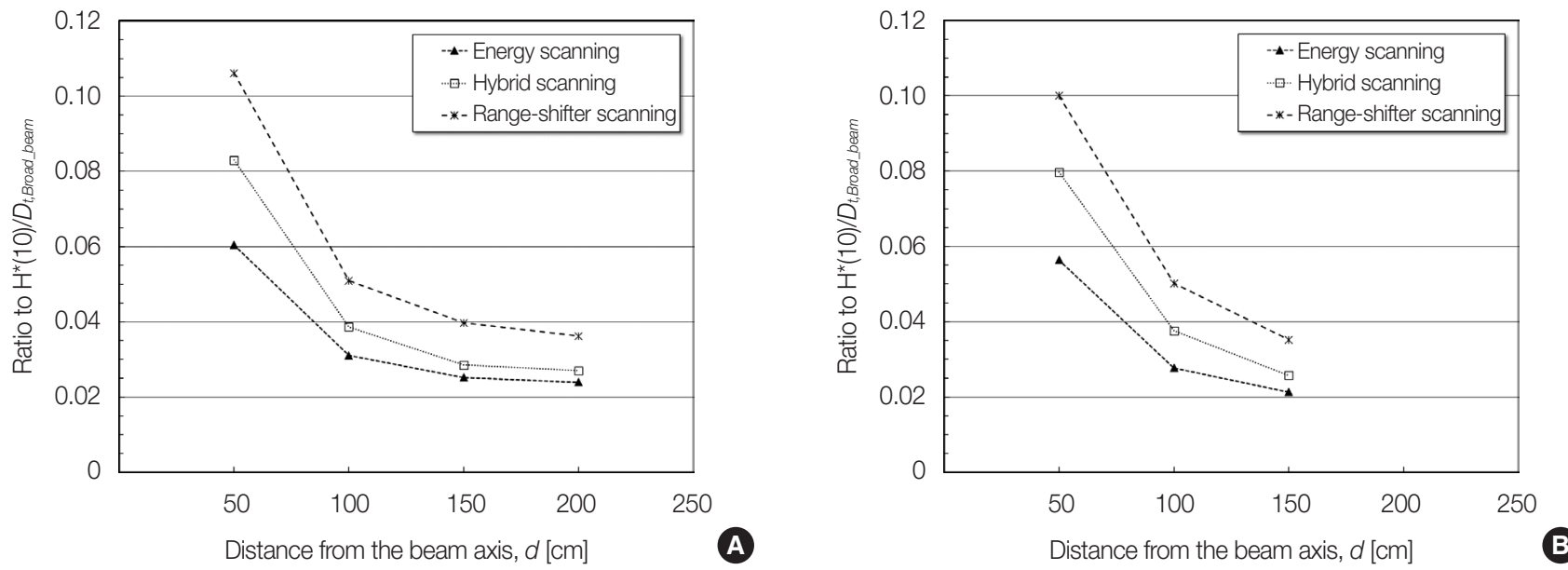

Fig. 3. Ratio of the measured $\mathrm{H}^{*}(10) / D_{t}$ with the scanning beam method to that with the broad beam method $\mathrm{H}^{*}(10) / D_{t, B \text { Broad beam: }}$ (A) $400 \mathrm{MeV} \cdot \mathrm{u}^{-1}$ and (B) $290 \mathrm{MeV} \cdot \mathrm{u}^{-1}$. 
lent $\mathrm{H}^{*}(10)$ in CIRT with various beam delivery methods for the 400 and $290 \mathrm{MeV} \cdot \mathrm{u}^{-1}$ primary carbon beams. The results were normalized by the treatment dose, $D_{t}$, defined as the clinical dose at the center of the spread-out Bragg peak. Fig. 3 presents the ratio of the measured $\mathrm{H}^{*}(10) / D_{t}$ with each scanning beam method to that with the broad beam method $\mathrm{H}^{*}(10) / D_{t, \text { Broad_beam }}$.

Although the neutron dose depends on the size and the volume of the irradiation target and beam energy, the measured $\mathrm{H}^{*}(10) / D_{t}$ ranged from 0.24 to $0.07 \mathrm{mSv} \cdot \mathrm{Gy}(\mathrm{RBE})^{-1}$ with the broad beam method and from 0.026 to $0.002 \mathrm{mSv} \cdot \mathrm{Gy}(\mathrm{RBE})^{-1}$ with the scanning beam method for the $400 \mathrm{MeV} \cdot \mathrm{u}^{-1}$ beam. No difference was observed in the tendency of the measured $\mathrm{H}^{*}(10) / D_{t}$ due to the differences in the beam energy and the beam delivery method. The measured $\mathrm{H}^{*}(10) / D_{t}$ with the scanning beam was less than approximately $10 \%$ of that with the broad beam at more than $50 \mathrm{~cm}$ from the beam axis when the irradiation target was the same. This ratio became smaller with the larger distances from the beam axis: from $4 \%$ to $2 \%$ of that with the broad beam at 150 and $200 \mathrm{~cm}$ with the scanning beam (Fig. 3). This tendency was derived from the presence of two types of secondary neutrons (i.e., internal and external neutrons). The external neutrons produced outside the patient by the interactions between the charged par-

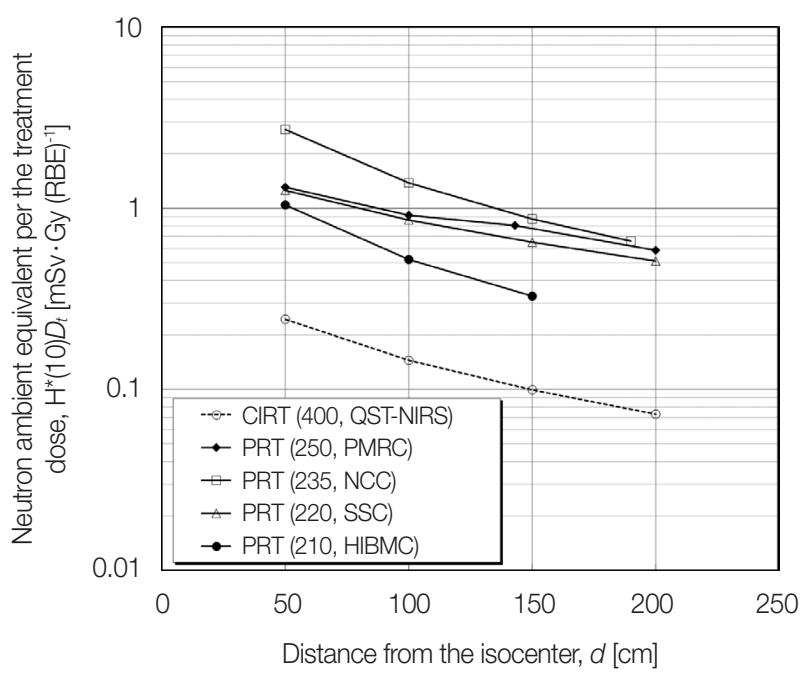

Fig. 4. Measured ambient dose equivalent in carbon-ion radiotherapy (CIRT) and proton radiotherapy (PRT) with the broad beam method. The legends show CIRT or PRT with the energy in $\mathrm{MeV} \cdot \mathrm{u}^{-1}$ and facility. QST-NIRS, National Institutes for Quantum and Radiological Science and Technology-National Institute of Radiological Sciences; HIBMC, Hyogo lon Beam Medical Center; NCCHE, National Canter Center Hospital East; SCC, Shizuoka Cancer Center; PMRC, Proton Medical Research Center at Tsukuba University. ticles and the beamline devices were greatly reduced using the scanning beam method compared to the broad beam method. Meanwhile, the internal neutrons produced in the patient were almost the same between the scanning and broad beam methods. Moreover, the internal neutrons were strongly produced in the forward direction because of the direct reaction process and deposited their energy more at the positions closer to the irradiation target. The angular dependency in the scanning beam method was investigated in [19].

The energy scanning technique can reduce the neutron dose most among the three techniques for the scanning beam because it needs a smaller number of primary carbon-ion particles due to fewer interactions with beamline devices, which leads to a lower neutron production compared with the other techniques.

\section{2) Comparison of the measured neutron ambient dose equivalents in CIRT and PRT}

Figs. 4 and 5 show the measured ambient dose equivalents in CIRT and PRT with the broad and scanning beam methods, respectively.

As shown in Fig. 4, the neutron doses in PRT with the broad beam method largely varied with the facility. This was derived from the differences of the operational beam setting and the beam delivery system design. However, for the broad beam, the neutron dose per treatment dose in CIRT was less than $30 \%$ of that in PRT, and the neutron dose in CIRT was clearly lower than that in PRT.

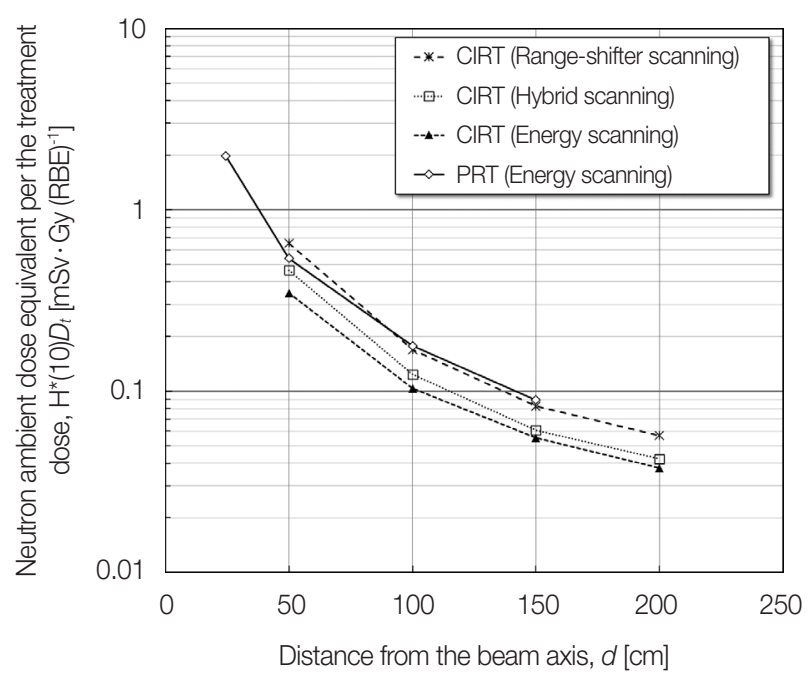

Fig. 5. Measured ambient dose equivalent in carbon-ion radiotherapy (CIRT) and proton radiotherapy (PRT) with the scanning beam method. The legends show the scanning technique. 


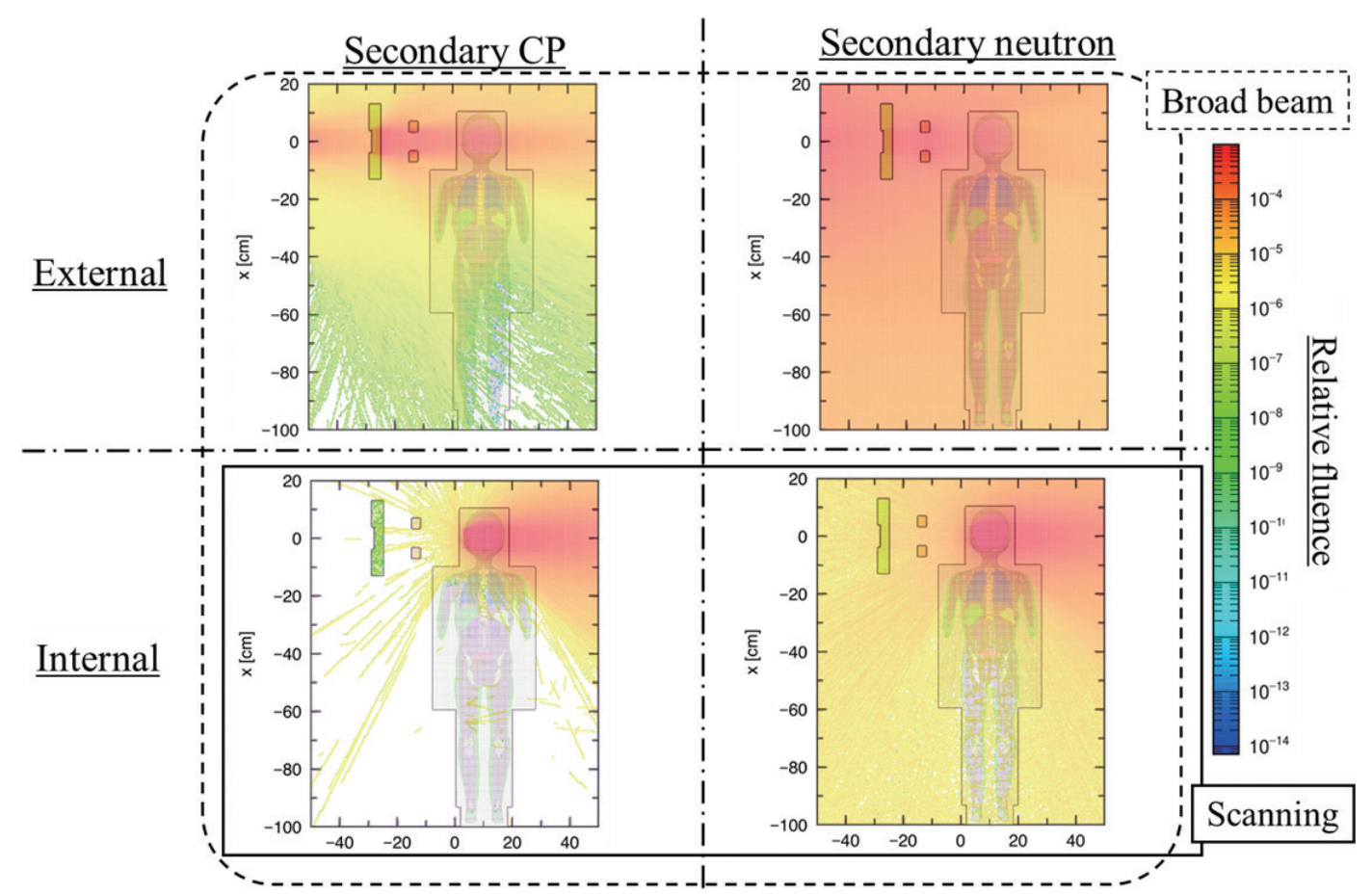

Fig. 6. Calculated particle tracks of the external/internal secondary charged particles (CPs)/secondary neutrons assuming pediatric brain tumor treatment.

In contrast, for the scanning beam with the energy scanning technique, the neutron dose per treatment dose in CIRT was lower than that in PRT, but the difference between CIRT and PRT was not as large as that for the broad beam method. These doses were measured under $90^{\circ}$ with respect to the beam axis. As mentioned before, the angular dependency in the scanning beam method with the energy scanning technique was investigated in [19]. The result indicated that the neutron dose in PRT showed a small angular dependency. In contrast, that in CIRT had a large angular dependency because heavier ions can strongly produce projectile and knockout neutrons in the forward direction. Therefore, the dose in CIRT can be greater than that in PRT as the angle decreased by less than $60^{\circ}$.

\section{Monte Carlo Calculations of the Out-of-Field Organ Doses in CIRT Assuming Prostate Cancer and Pediatric Brain Tumor Treatments}

1) Contribution of the secondary neutrons to the out-of-field organ dose

Fig. 6 illustrates the calculated particle tracks of the external and internal charged particles (CPs) and external and internal neutrons with the PHITS code to easily understand their distribution. As mentioned before, secondary CPs cause the out-of-field dose in CIRT and the secondary neu- trons. The internal and external CPs and the internal neutrons were distributed along the beam axis, while the external neutrons were widely distributed, showing that the internal and external CPs and the internal neutrons contributed to the dose near the target volume, while the external neutrons contributed to the whole-body dose.

Fig. 7 depicts the partial contributions to the total dose equivalent of the secondary and internal neutrons for the selected organs in CIRT for prostate and pediatric brain cancers. The contribution of the secondary neutrons to the total dose was approximately $30 \%$ at $10 \mathrm{~cm}$ from the target and increased up to higher than $80 \%$ along with the distance from the target. The contribution for prostate cancer treatment was higher than that for pediatric brain cancer treatment. The reason for this should be derived from the difference of the energy of the primary carbon beam: $400 \mathrm{MeV} \cdot \mathrm{u}^{-1}$ in $13 \mathrm{frac}-$ tions and $290 \mathrm{MeV} \cdot \mathrm{u}^{-1}$ in three fractions for prostate cancer treatment and only $290 \mathrm{MeV} \cdot \mathrm{u}^{-1}$ for pediatric brain cancer treatment. Higher-energy primary carbon ions can produce more secondary neutrons, while the doses due to the secondary CPs by 290 and $400 \mathrm{MeV} \cdot \mathrm{u}^{-1}$ carbon ions are not really basically different. In contrast, the contributions of the internal neutrons did not dramatically change depending on the distance from the target. Internal neutrons were produced 


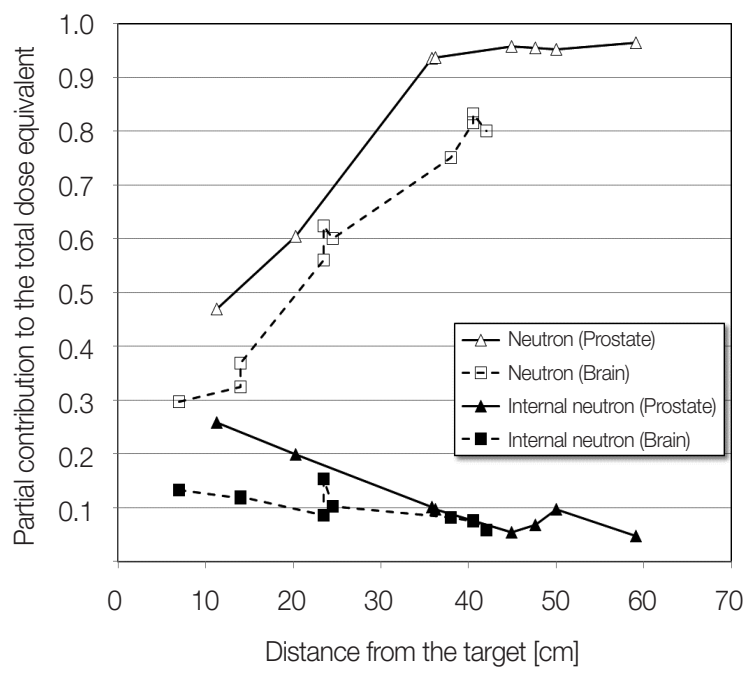

Fig. 7. Partial contributions to the total dose equivalent due to neutrons and internal neutrons for selected organs. "Prostate" and "Brain" indicates the results for prostate cancer treatment and pediatric brain cancer, respectively. The lines are drawn to guide the eye.

in the forward direction, but the partial contribution did not increase near the target because the secondary CPs were also in the forward direction. The contribution of the internal neutrons in prostate cancer treatment was slightly higher than that in pediatric brain cancer treatment, but we expected that the reason for this is that a patient-specific collimator and a range compensator set in the front of a patient, in which external secondary neutrons and CPs were additionally produced, were not be considered in the prostate cancer treatment evaluation.

Although the secondary CPs are not shown in Fig. 7, almost all the rest, except for the neutrons, were caused by the secondary CPs. The secondary CPs contributed more to the total dose for organs closer to the target. We focused on the secondary neutrons in this review, but note that accurately evaluating the secondary CPs, especially near the target, is essential to the dose assessment of the evaluated patient.

\section{2) Calculated out-of-field organ doses in CIRT}

Fig. 8 shows the out-of-field organ dose equivalents per treatment dose as a function of the distance from the center of the target volume compared to those in IMRT. The lower bound shown in AAPM TG-158 (American Association of Physicists in Medicine Task Group). was derived from many research results from various IMRT treatments representing different treatment sites, treatment energies (6-18 MV) and delivery systems based on the measurement and simulation

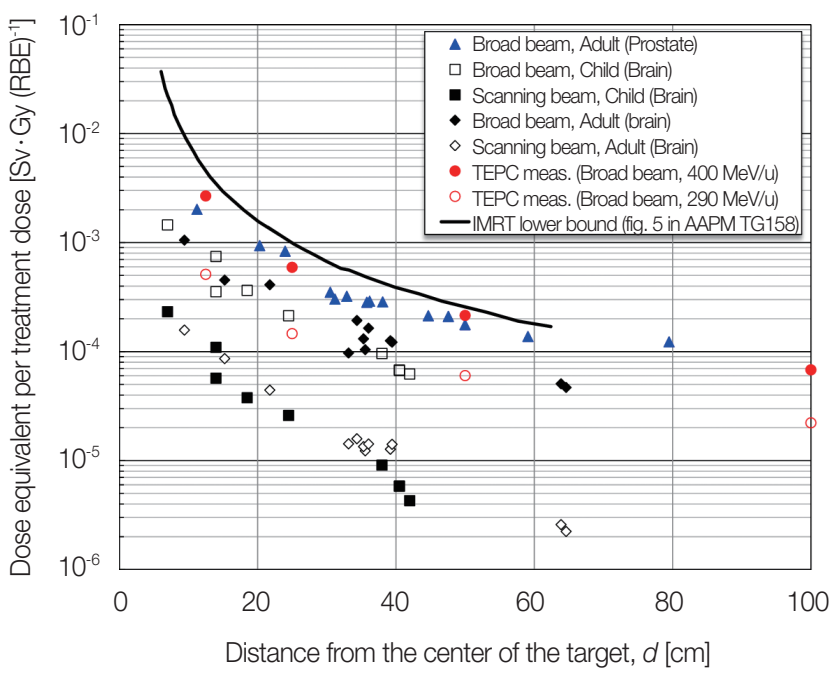

Fig. 8. Out-of-field organ doses in carbon-ion radiotherapy for prostate and brain cancer. "TEPC meas" indicates the measured results with a tissue equivalent proportional counter [14]. "IMRT lower bound" indicates lower bound that describes the empirical range of doses associated with different intensity-modulated radiotherapy techniques [12]. AAPM TG-158, American Association of Physicists in Medicine Task Group.

[12]. When energies of photon beams exceed approximately $10 \mathrm{MV}$, neutrons are produced in the head of the accelerator in theory, but the lower bound did not include the neutron dose. But this does not change the lower bound because the lower bound was derived from the 6-MV IMRT. In contrast, the dose in CIRT included the contributions from all the radiation species. The dose in CIRT decreased with the distance to the target in a similar manner to the tendency in IMRT and was less than the lower bound describing the empirical range of the doses associated with different IMRT techniques. The results for the broad and scanning beam methods for brain cancer treatments are shown in Fig. 8, indicating that the scanning beam method can reduce the dose by less than $16 \%$ compared to the broad beam method. These results also showed that the out-of-field dose depends only on the distance from the target, regardless of the phantom size (patient's body size), and pediatric patients are prone to receive an out-of-field dose due to their small body. Nevertheless, the neutron production strongly depends on the beam energy, and a small body should lead to less neutron production by the primary beam.

In addition, the experimental results measured with a tissue equivalent proportional counter and a homogeneous water phantom for the 290 and $400 \mathrm{MeV} \cdot \mathrm{u}^{-1}$ carbon beams with the broad beam method were shown together. The val- 
ues in CIRT with the broad beam method for prostate and brain cancer were very close to those measured for the 400 and $290 \mathrm{MeV} \cdot \mathrm{u}^{-1}$ carbon beams, respectively. This result indicates that simple experiments with homogeneous materials using a beam with energy assuming the treatment irradiation may become a practical tool for estimating the out-offield organ doses.

\section{Conclusion}

This review introduced investigations on the unwanted neutron dose in CIRT. The measurements of the neutron ambient dose equivalents under $90^{\circ}$ to the beam axis in CIRT with the broad beam method clearly showed that the neutron dose per treatment dose in CIRT was lower than that in PRT. For the scanning beam with the energy scanning technique, the neutron dose per treatment dose in CIRT was lower than that in PRT, but the difference between CIRT and PRT was not as large as that for the broad beam method. Our measured results indicated that the neutron dose in PRT may be lower than that in CIRT as the angle decreased by less than $60^{\circ}$ because of the less angular dependence in PRT. Moreover, the out-of-field organ doses by all the radiation species in CIRT were evaluated with the PHITS code and found to decrease with the distance to the target and less than the lower bound in IMRT shown in AAPM TG-158.

The evaluation of the out-of-field doses is important from the viewpoint of secondary cancer risk after radiotherapy. Secondary neutrons are considered as the major source in CIRT, especially in the distant area from the target volume. However, the dose level in CIRT is similar or lower than that in PRT and IMRT, even if the contributions from all radiation species are included in the evaluation. In particular, CIRT with the scanning beam method with the energy scanning technique can greatly reduce the unwanted out-of-field dose and serve as a promising radiotherapy modality for pediatric treatment from the viewpoint of out-of-field exposure.

\section{Conflict of Interest}

No potential conflict of interest relevant to this article was reported.

\section{Author Contribution}

Conceptualization: Yonai S. Data curation: Yonai S, Matsu- moto S. Visualization: Yonai S, Matsumoto S. Writing - original draft: Yonai S. Writing - review \& editing: Yonai S, Matsumoto S. Investigation: Yonai S.

\section{References}

1. Tsujii H, Kamada T. A review of update clinical results of carbon ion radiotherapy. Jpn J Clin Oncol. 2012;42:670-685.

2. Durante M. New challenges in high-energy particle radiobiology. Br J Radiol. 2014;87:20130626.

3. Halperin EC. Particle therapy and treatment of cancer. Lancet Oncol. 2006;7:676-685.

4. Schulz-Ertner D, Tsujii H. Particle radiation therapy using proton and heavier ion beams. J Clin Oncol. 2007;25:953-964.

5. Pompos A, Durante M, Choy H. Heavy ions in cancer therapy. JAMA Oncol. 2016;2:1539-1540.

6. Hall EJ. Intensity-modulated radiation therapy, protons, and the risk of second cancers. Int J Radiat Oncol Biol Phys. 2006;65:1-7.

7. Brenner DJ, Hall EJ. Secondary neutrons in clinical proton radiotherapy: a charged issue. Radiother Oncol. 2008;86:165-170.

8. Newhauser WD, Durante M. Assessing the risk of second malignancies after modern radiotherapy. Nat Rev Cancer. 2011;11: 438-448.

9. Stokkevag CH, Schneider U, Muren LP, Newhauser W. Radiationinduced cancer risk predictions in proton and heavy ion radiotherapy. Phys Med. 2017;42:259-262.

10. National Council on Radiation Protection and Measurements. Second primary cancers and cardiovascular disease after radiation therapy (Report No. 170). Bethesda, MD: National Council on Radiation Protection and Measurements; 2011.

11. Farah J, Mares V, Romero-Exposito M, Trinkl S, Domingo C, Dufek $\mathrm{V}$, et al. Measurement of stray radiation within a scanning proton therapy facility: EURADOS WG9 intercomparison exercise of active dosimetry systems. Med Phys. 2015;42:2572-2584.

12. Kry SF, Bednarz B, Howell RM, Dauer L, Followill D, Klein E, et al. AAPM TG 158: Measurement and calculation of doses outside the treated volume from external-beam radiation therapy. Med Phys. 2017;44:e391-e429.

13. Yonai S, Matsufuji N, Kanai T, Matsui Y, Matsushita K, Yamashita $\mathrm{H}$, et al. Measurement of neutron ambient dose equivalent in passive carbon-ion and proton radiotherapies. Med Phys. 2008; 35:4782-4792.

14. Yonai S, Kase Y, Matsufuji N, Kanai T, Nishio T, Namba M, et al. Measurement of absorbed dose, quality factor, and dose equivalent in water phantom outside of the irradiation field in passive carbon-ion and proton radiotherapies. Med Phys. 2010;37:40464055.

15. Yonai S, Matsufuji N, Namba M. Calculation of out-of-field dose distribution in carbon-ion radiotherapy by Monte Carlo simula- 
tion. Med Phys. 2012;39:5028-5039.

16. Yonai S, Furukawa T, Inaniwa T. Measurement of neutron ambient dose equivalent in carbon-ion radiotherapy with an active scanned delivery system. Radiat Prot Dosimetry. 2014;161:433436.

17. Yonai S, Matsufuji N, Akahane K. Monte Carlo study of out-offield exposure in carbon-ion radiotherapy with a passive beam: organ doses in prostate cancer treatment. Phys Med. 2018;51: 48-55.

18. Matsumoto S, Yonai S, Bolch WE. Monte Carlo study of out-offield exposure in carbon-ion radiotherapy: Organ doses in pediatric brain tumor treatment. Med Phys. 2019;46:5824-5832.

19. Matsumoto S, Yonai S. Evaluation of neutron ambient dose equivalent in carbon-ion radiotherapy with energy scanning. Radiat Prot Dosimetry. 2020;191:310-318.

20. Olsher RH, Hsu HH, Beverding A, Kleck JH, Casson WH, Vasilik DG, et al. WENDI: an improved neutron rem meter. Health Phys. 2000;79:170181.

21. Schneider U, Agosteo S, Pedroni E, Besserer J. Secondary neutron dose during proton therapy using spot scanning. Int J Radiat Oncol Biol Phys. 2002;53:244-251.

22. Kawachi K, Kanai T, Matsuzawa H, Inada T. Three dimensional spot beam scanning method for proton conformation radiation therapy. Acta Radiol Suppl. 1983;364:81-88.

23. Haberer T, Becher W, Schardt D, Kraft G. Magnetic scanning sys- tem for heavy ion therapy. Nucl Instrum Methods Phys Res A. 1993;330:296-305.

24. Furukawa T, Hara Y, Mizushima K, Saotome N, Tansho R, Saraya Y, et al. Development of NIRS pencil beam scanning system for carbon ion radiotherapy. Nucl Instrum Methods Phys Res B. 2017;406:361-367.

25. International Electrotechnical Commission. Medical electrical equipment - Part 2-64: Particular requirements for the basic safety and essential performance of light ion beam medical electrical equipment. Geneva, Switzerland: International Electrotechnical Commission; 2014. IEC 60601-2-64:2014.

26. Sato T, Iwamoto Y, Hashimoto S, Ogawa T, Furuta T, Abe SI, et al. Features of particle and heavy ion transport code system (PHITS) version 3.02. J Nucl Sci Technol. 2018;55:684-690.

27. Menzel HG, Clement C, DeLuca P. ICRP Publication 110: Realistic reference phantoms: an ICRP/ICRU joint effort. A report of adult reference computational phantoms. Ann ICRP. 2009;39: $1-164$.

28. Lee C, Lodwick D, Hurtado J, Pafundi D, Williams JL, Bolch WE. The UF family of reference hybrid phantoms for computational radiation dosimetry. Phys Med Biol. 2010;55:339-363.

29. International Commission on Radiological Protection. 1990 Recommendations of the International Commission on Radiological Protection. Ann ICRP. 1991;21:1-201. 